\title{
Thrombin Induces Long-Term Potentiation of Reactivity to Afferent Stimulation and Facilitates Epileptic Seizures in Rat Hippocampal Slices: Toward Understanding the Functional Consequences of Cerebrovascular Insults
}

\author{
Nicola Maggio, ${ }^{1}$ Efrat Shavit, ${ }^{2}$ Joab Chapman, ${ }^{2}$ and Menahem Segal ${ }^{1}$ \\ ${ }^{1}$ Department of Neurobiology, The Weizmann Institute, 76100 Rehovot, Israel, and ${ }^{2}$ Departments of Physiology and Pharmacology and Neurology, Sackler \\ Faculty of Medicine, Tel Aviv University, Tel Aviv 69978, Israel
}

The effects of thrombin, a blood coagulation serine protease, were studied in rat hippocampal slices, in an attempt to comprehend its devastating effects when released into the brain after stroke and head trauma. Thrombin acting through its receptor, protease-activated receptor 1 (PAR1), produced a long-lasting enhancement of the reactivity of CA1 neurons to afferent stimulation, an effect that saturated the ability of the tissue to undergo tetanus-induced long-term potentiation. This effect was mediated by activation of a PAR1 receptor, because it was shared by a PAR1 agonist, and was blocked by its selective antagonist. An independent effect of thrombin involved the lowering of the threshold for generating epileptic seizures in CA3 region of the hippocampus. Thus, the experiments in a slice mimicked epileptic and cognitive dysfunction induced by thrombin in the brain, and suggest that these effects are mediated by activation of the PAR1 receptor.

Key words: hippocampus; thrombin; LTP; seizure; PAR1; cerebrovascular insult

\section{Introduction}

Cerebrovascular injuries induced by either ischemia or hemorrhage manifest themselves by short- and long-term loss of numerous brain functions as well as by induction of epileptic seizures (Camilo and Goldstein, 2004; de Haan et al., 2006; Henon et al., 2006). The main pathogenic mechanisms proposed for such brain dysfunctions include hypoxia, glutamate toxicity, electrolyte dysfunction, and eventual cell death. An additional putative source of complication after a vascular trauma consists of the exposure of brain tissue to blood constituents (Hua et al., 2007). In particular, thrombin, a serine protease critical in the clotting cascade, may come in direct contact with neuronal tissue and be involved in the production of seizures (Gingrich and Traynelis, 2000). The highest levels of thrombin activity in the brain are produced by intracerebral hemorrhagic stroke (ICH) and subarachnoid hemorrhage (SAH), and in both cases, there is a high incidence of epileptic activity during the initial phases (Lin et al., 2003; Claassen et al., 2007). In both types of hemorrhage, the early rate of seizures (20-30\%) is much higher than the rate of chronic epilepsy (2\%), suggesting an immediate effect of coagulation factors such as thrombin in causing early seizures. This has

\footnotetext{
Received Aug. 12, 2007; revised Dec. 2, 2007; accepted Dec. 4, 2007.

This work was supported by a grant from the Nella and Leon Benoziyo Center for Neurological Diseases and The Weizmann Institute.

Correspondence should be addressed to Menahem Segal at the above address. E-mail: menahem.segal@weizmann.ac.il.

DOI:10.1523/JNEUROSCI.3665-07.2008

Copyright $\odot 2008$ Society for Neuroscience $\quad$ 0270-6474/08/280732-05\$15.00/0
}

been directly supported in a rat model of ICH in which active thrombin injected into rat brains resulted in both electrographic and clinical seizures (Lee et al., 1997). Current studies suggest that thrombin has a range of effects on evoked potentials and spontaneous activity (Lee et al., 1997, 2007; Gingrich et al., 2000; Lee and Haydon, 2007). Nevertheless, the effects of thrombin on neuronal tissue, as well as its relevance to the long-term cognitive and neurological consequences of brain vascular insults, are not entirely clear. We now propose that thrombin, acting on its receptor, protease-activated receptor 1 (PAR1), has marked effects on the production of long-term potentiation (LTP) in responses to afferent stimulation and that it enhances the sensitivity to epileptic seizures in brain slices. These actions of thrombin may underlie some neurological consequences of acute cerebrovascular insults.

\section{Materials and Methods}

The methods are detailed previously (Maggio and Segal, 2007). Briefly, 2-month-old male Wistar rats were rapidly decapitated, and $350 \mu \mathrm{m}$ transverse or horizontal hippocampal slices were used. Slices were incubated for $1.5 \mathrm{~h}$ in a humidified, carbogenated $\left(5 \% \mathrm{CO}_{2}\right.$ and $\left.95 \% \mathrm{O}_{2}\right)$ gas atmosphere at $33 \pm 1{ }^{\circ} \mathrm{C}$ and were perfused with artificial CSF [containing (in mM) $124 \mathrm{NaCl}, 2 \mathrm{KCl}, 26 \mathrm{NaHCO}_{3}, 1.24 \mathrm{KH}_{2} \mathrm{PO}_{4}, 2.5 \mathrm{CaCl}_{2}, 2$ $\mathrm{MgSO}_{4}$, and 10 glucose, $\mathrm{pH} 7.4$ ] in a standard interface chamber. Recordings were made with a glass pipette containing $0.75 \mathrm{M} \mathrm{NaCl}(4 \mathrm{M} \Omega$ ) placed in the stratum radiatum CA1 or CA3. Stimulation was evoked using a Master 8 pulse stimulator (A.M.P.I., Jerusalem, Israel) and was delivered through two sets of bipolar nichrome electrodes placed on either side of the recording electrode such that two independent stimulation channels were used for each slice. LTP was induced by high- 
A

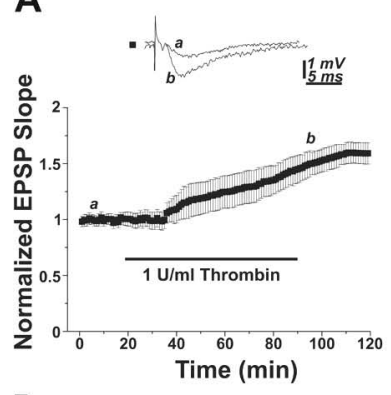

D

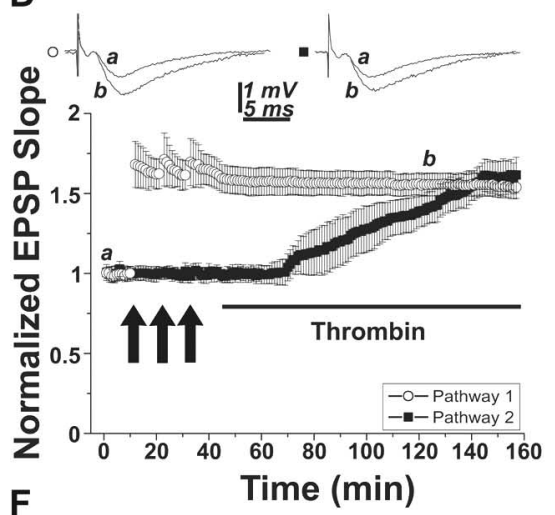

$\mathbf{F}$
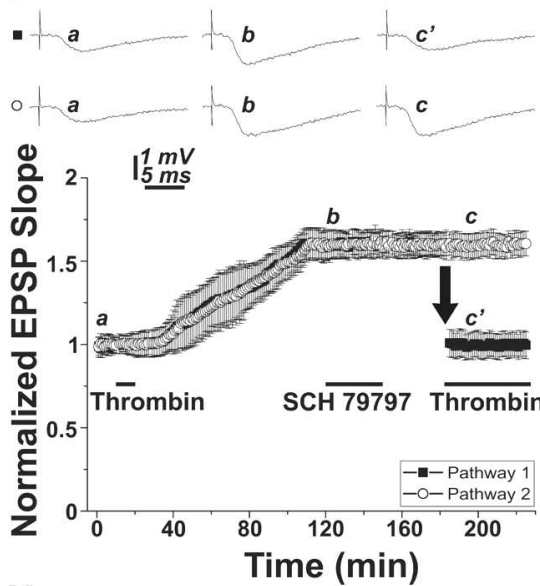

H
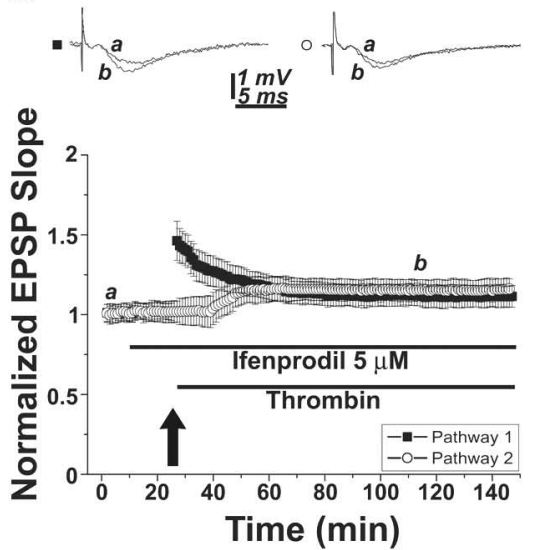

C

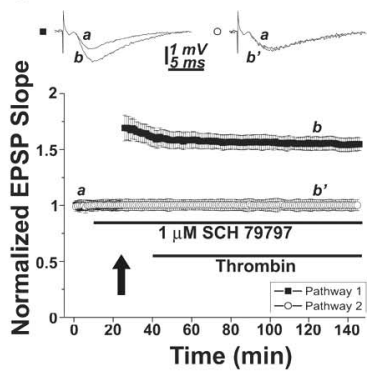

$E$

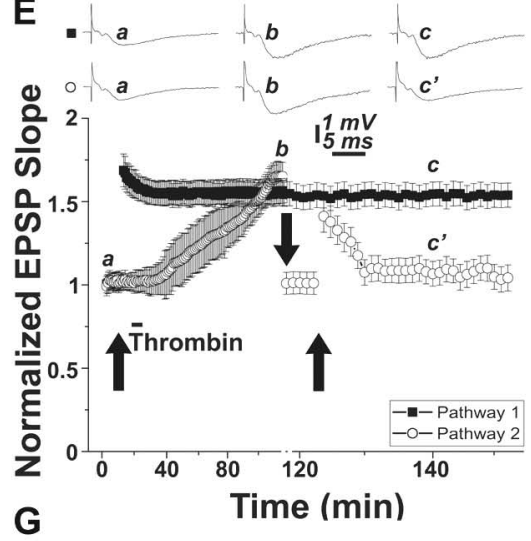

G

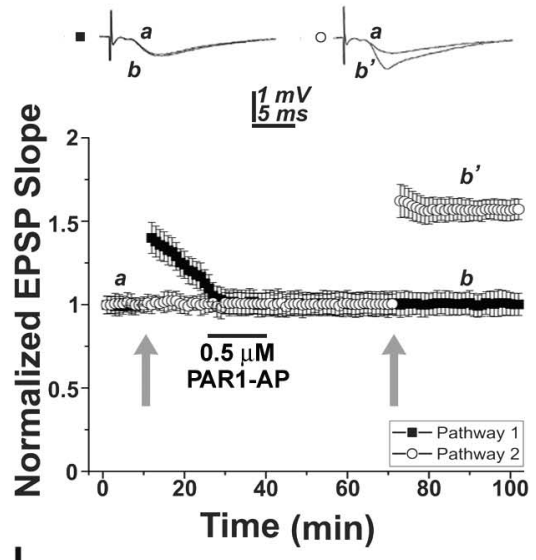

I

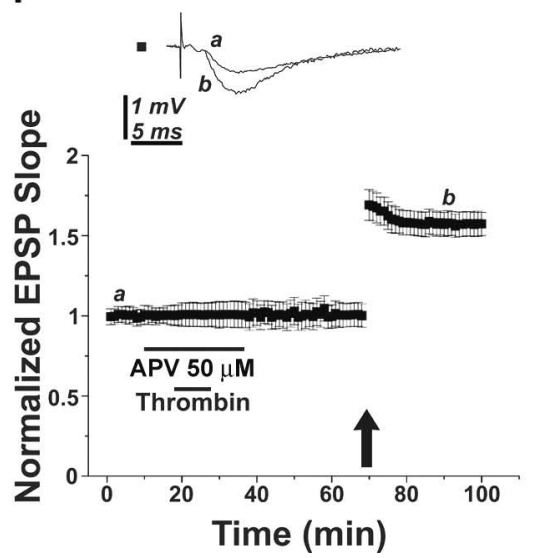

Figure 1. Thrombin induces long-term potentiation of reactivity to afferent stimulation and saturates tetanus-induced LTP. $\boldsymbol{A}$, $\boldsymbol{B}$, Application of thrombin $(1 \mathrm{U} / \mathrm{ml} ; \boldsymbol{A})$ as well as the PAR1 receptor agonist, PAR1-AP $(\boldsymbol{B})$, produces a gradual increase in population EPSP in stratum radiatum CA1, without affecting population presynaptic volleys (top traces). $C$, Thrombin-induced LTP is blocked by the PAR1 receptor antagonist SCH79797, which does not affect tetanic LTP. D, Thrombin induces LTP in a naive pathway without affecting an already saturated pathway, which was previously exposed to three tetanic stimulations. $E$, A short (10 min) application of thrombin produces a saturating LTP; a tetanic stimulation to the same pathway, adjusted to submaximal level (downward arrow), is able to produce a short-term but not a long-term potentiation. $\boldsymbol{F}$, 0 nce thrombin-induced LTP has been

frequency stimulation consisting of 100 pulses at twice the test intensity, delivered at a frequency of $100 \mathrm{~Hz}$ (HFS; $100 \mathrm{~Hz}, 1 \mathrm{~s}$ ). Before applying the tetanic stimulation, baseline values were recorded at a frequency of $0.033 \mathrm{~Hz}$. Responses were digitized at $5 \mathrm{kHz}$ and stored on a computer. Off-line analysis and data acquisition were performed using the LTP Program (Anderson and Collingridge, 2001) and Axoclamp software.

All numerical data are expressed as mean \pm SEM, and EPSP slope changes after tetanic stimulation were calculated with respect to baseline. There were no systematic differences in the magnitudes of the baseline responses in the different conditions. All values reported refer to $30 \mathrm{~min}$ before tetanic stimulation. Unless otherwise indicated, statistical evaluations were performed by applying a Student's $t$ test for paired and unpaired data, as the case may be (Origin 6.0). $p$ values of $<0.05$ were considered a significant difference between means.

\section{Results}

Exposure to thrombin (applied in the perfusion medium at a concentration of 1 $\mathrm{U} / \mathrm{ml}$ ) produced a gradual increase in population EPSP recorded in stratum radiatum of region CA1 of the hippocampus. This gradual change was specific to the EPSP, because no parallel change was noticed in the presynaptic volley produced in response to the stimulation (Fig. $1 A$ ). The increase in EPSP slope rose gradually over $40 \mathrm{~min}$ of recording, reached a plateau level, which was $51 \%$ above control $(n=9$ slices; $1.51 \pm 0.09 ; p<0.01$ ), and was persistent after washout of thrombin for as long as recording was made (up to $3 \mathrm{~h}$; see below).

A very similar, slow-rising, persistent increase in population EPSP was seen after bath application of $15 \mu \mathrm{M}$ PAR1-AP (SFLLRN), a PAR1 receptor agonist $(n=9$ slices; $1.56 \pm 0.09 ; p<0.01)$. To verify that the effect of thrombin is mediated through

$\leftarrow$

established, it cannot be blocked by later application of SCH79797, nor could it be further potentiated by a second sustained application of the drug, applied to an adjusted response level (downward arrow). G, Low concentration of PAR1-AP amplifies the ability of a short tetanic stimulation to produce LTP. Short tetanic stimulation applied before PAR1-AP was able to produce a short-lasting but no long-lasting potentiation. PAR1-AP at a low concentration $(0.5 \mu \mathrm{m})$ did not affect responses to the stimulation, but was able to convert a short into a long-lasting potentiation to short stimulation applied later. $\boldsymbol{H}$, Thrombin-induced LTP is partially blocked by application of the NR2B antagonist, ifenprodil, as is the potentiation produced by the tetanic stimulation. $I$, The NMDA antagonist APV totally blocks thrombin-induced LTP, but after washout of the drug, tetanic LTP is completely normal. Averaged EPSP slopes are plotted versus time. Representative traces at indicated times $(a, b)$ are shown on top of each section. Upward arrows indicate the time of HFS. 
activation of a genuine PAR1 receptor, the selective antagonist SCH79797 was used. In these experiments, we introduced a second stimulating electrode to produce LTP by tetanic stimulation. The PAR1 antagonist SCH79797 (Tocris, Bristol, UK) ( $1 \mu \mathrm{M}$ prepared from $50 \mathrm{~mm}$ stock DMSO solution) had no effect on tetanic LTP (Fig. 1C) $(n=9 ; 1.55 \pm 0.06$ and $1.00 \pm 0.05)$, but the response to thrombin, tested in the nontetanized pathway, was completely abolished. These experiments indicate that the effect of thrombin is upstream to the tetanic stimulation in that its blockade did not affect the ability of the neurons to increase their responses to the afferent stimulation.

We next examined the possibility that thrombin and tetanic stimulation share a common path for enhancing reactivity to afferent stimulation. The following experiments were conducted: in the first, one pathway was tetanized intensely using three 100 $\mathrm{Hz}$ stimulation trains, and the other one was not tetanized. Subsequently, the slices were exposed to thrombin. Under these conditions, only the previously nontetanized pathway reacted to thrombin by the typical long-term increase in reactivity, indicating that the other one was already saturated (Fig. 1D) $(n=7$; $1.49 \pm 0.10$ and $1.55 \pm 0.07)$. The reverse experiment was then conducted; one pathway in the slice was tetanized and the slice exposed to thrombin. When the response reached asymptote, stimulation intensity at the nontetanized pathway was reduced to the level before drug/tetanus application, and a tetanic stimulation was then applied. Under this condition, there was only a transient elevation of the responses so as to produce short-term potentiation, which was reduced back to baseline level within 5-10 min, indicating that the slice was able to express short-term but not long-term potentiation after exposure to thrombin.

Because thrombin is a fairly large molecule, not likely to wash out readily, we examined the possibility that a continuous presence of thrombin in the slice and not a long-lasting effect of the protein causes the prolonged enhanced reactivity. Two experiments were conducted to examine this possibility. In one, shown above, thrombin was applied for only a short duration and washed out extensively. In these cases, the gradual increase in the response was still evident despite the likelihood that thrombin was no longer present in the slice. In another series of experiments (Fig. $1 F$ ), thrombin was applied for a short interval, and the enhanced response was followed by exposure to the SCH79797 compound. In these experiments, once the response was elevated by thrombin, the antagonist was no longer able to reverse this effect. This indicates that the continuous presence of thrombin is not necessary for the production of the enhanced response, which can be triggered by a short exposure to the compound.

Finally, we examined the possibility that a tetanic stimulation interacts with thrombin to produce a long-lasting LTP (Fig. 1G) (six slices). This experiment was conducted with the PAR1 agonist, PAR1-AP. In preliminary experiments, the threshold dose of PAR1-AP $(0.5 \mu \mathrm{M})$ that by itself does not produce a change in response to the afferent stimulation was determined. A short tetanic stimulation ( 25 pulses at $100 \mathrm{~Hz}$ ), that by itself produces only a short-lasting potentiation, was then applied. When the short tetanic stimulation was applied to one of two pathways after exposure to the low dose of PAR1-AP, a full-blown LTP was generated, only in this pathway, and not in the other one tetanized before exposure to PAR1-AP. This indicates that a subthreshold concentration of PAR1-AP can still enhance the ability of a tetanic stimulation to produce a long-lasting potentiation.

If indeed thrombin shares a downstream mechanism with the conventional LTP, it should be sensitive to drugs that depress
LTP. There are many ways to suppress LTP, studied extensively previously. In one set of experiments, we examined the effects of ifenprodil, an NR2B receptor antagonist, which has a controversial effect on LTP (Liu et al., 2004; Li et al., 2007). Curiously, in our hands ifenprodil $(5 \mu \mathrm{M})$ suppressed the ability to express tetanic LTP (down to $1.21 \pm 0.06$ of control; $n=7$ slices) (Fig. $1 \mathrm{H}$ ), and in these same slices the effects of thrombin were also reduced down to $1.23 \pm 0.054$ of controls in presence of the drug. In a second set of experiments, thrombin was applied in the presence of the general NMDA receptor antagonist APV $(50 \mu \mathrm{M})$. Under these conditions, thrombin was unable to produce LTP ( $1.02 \pm 0.08$ after 20 min from thrombin washout; $n=6$ slices) (Fig. 1I); however, washout of both drugs did not impair the later ability of the slice to express tetanic LTP. These experiments indicate that thrombin shares the same downstream mechanisms involving the NMDA receptor, which is activated by the tetanic stimulation to produce LTP.

These experiments demonstrate that thrombin is likely to saturate the LTP-generating mechanism in region CA1 of the rat hippocampus in a manner that is pathway independent, to the extent that the hippocampus is unable to produce LTP to a new tetanic stimulation.

To examine the possibility that this action of thrombin is restricted to region CA1, we studied its effect on responses evoked in CA3 to afferent stimulation applied in stratum radiatum, which activates the commissural/association pathway. A similar long-lasting enhancement of reactivity to afferent stimulation was found in CA3, indicating that this type of LTP is not unique to region CA1 (supplemental Fig. 1, available at www.jneurosci.org as supplemental material) ( $n=9$ slices for each experiment).

Thrombin has been suggested to trigger the production of epileptic seizures in vivo (Xi et al., 2003) through a yet unknown mechanism, but no evidence for such an action was seen in our experiments; i.e., there was no sign of multiple population spikes or spontaneous interictal spikes in either CA3 or CA1 regions. The only indication that thrombin causes some change in spontaneous activity was seen in recordings from CA3 pyramidal layer. In these (Fig. 2A), application of thrombin or the PAR1 agonist produced a long-lasting increase in spontaneous firing of CA3 but not CA1 neurons ( $n=9$ slices for each of the pharmacology). To examine the possibility that thrombin facilitates the effects of other conditions known to produce epileptic dischargelike activity in the slice, we elevated extracellular $\mathrm{K}^{+}$concentration by $4 \mathrm{~mm}$ above control (3.2 $\mathrm{mm}$ ) level. In normal slices, addition of $4 \mathrm{mM} \mathrm{K}^{+}$did not produce any noticeable spontaneous seizures ( $n=9$ slices), which were clearly seen if extracellular level of $\mathrm{K}^{+}$was raised by $15 \mathrm{~mm}$ ( $n=7$ slices) (Fig. $2 B$ ) (Jensen and Yaari, 1997). Likewise, $500 \mu \mathrm{M}$ but not $100 \mu \mathrm{M}$ glutamate could produce spontaneous seizure-like activity in the slice (Fig. $2 B)$ ( $n=9$ slices) (Sun et al., 2001). However, in presence of thrombin, the lower concentration of $\mathrm{K}^{+}(4 \mathrm{~mm})$ and glutamate $(100 \mu \mathrm{M})$ did produce seizure-like activity. This activity was mediated by the PAR1 agonist action of thrombin ( $n=7$ slices), because it was mimicked by PAR1-AP ( $n=7$ slices) and blocked by the SCH79797 compound ( $n=7$ slices) (Fig. $2 C$ ). Interestingly, the facilitating action of thrombin on the production of seizure-like activity was not blocked by ifenprodil ( $n=6$ slices) or by APV $(n=6$ slices), which did attenuate or block, respectively, the thrombininduced LTP (above), indicating that this action is not shared by the NMDA receptor activated to produce LTP.

Finally, seizure-like activity was detected both in CA3 and CA1 regions of the hippocampal slice. Because other studies have shown that epilepsy is generated in CA3 and spreads to CA1 
A

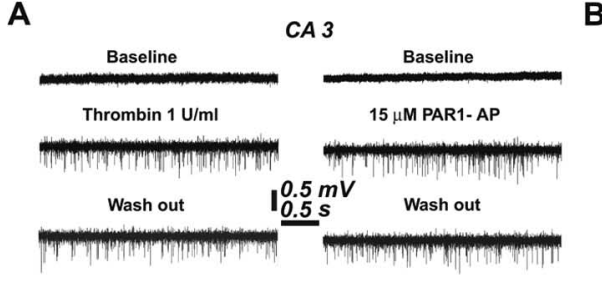

C

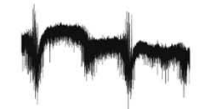

$4 \mathrm{mM} \mathrm{KCl}+$ Thrombin

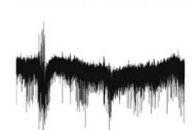

$100 \mu \mathrm{M}$ Glut + Thrombin
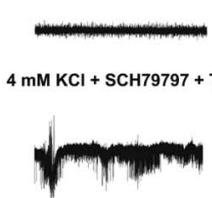

$4 \mathrm{mM} \mathrm{KCl}+$ Ifenprodil + Thrombin

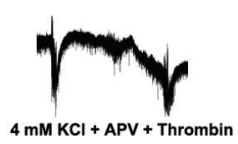

Figure 2. Thrombin facilitates the onset of seizure-like activity in hippocampal neurons. $\boldsymbol{A}$, Thrombin as well as PAR1-AP produces a sustained increase in spontaneous spike activity of single neurons in region CA3. Such an effect is not seen in CA1 region (see below, $\boldsymbol{D}$ ). $\boldsymbol{B}$, Increasing extracellular concentrations of $\mathrm{K}^{+}$by $4 \mathrm{~mm}$ or exposure to $100 \mu \mathrm{m}$ glutamate does not affect spontaneous activity, but a larger increase, by $15 \mathrm{~mm} \mathrm{KCl}$ or $500 \mu \mathrm{m}$ glutamate, produces synchronized seizure-like activity. $\boldsymbol{C}$, Thrombin or PAR1-AP facilitates the onset of seizure-like activity in the presence of below-threshold concentrations of $\mathrm{K}^{+}$or glutamate $(100 \mu \mathrm{M})$. Those seizure-like activities are blocked by PAR1 receptor antagonist, but not by NR2B or NMDA antagonists. D, The onset of seizure-like activity in CA1 depends on its anatomical connections with CA3; in intact hippocampus (middle trace), seizure activity is seen, but is absent when the connection between CA 3 and CA1 is severed (bottom trace).

(Jensen and Yaari, 1988; Traynelis and Dingledine, 1988), we examined the possibility that a locus of seizure activity is also resident in CA1. To this end, a vertical cut between CA3 and CA1 was made and activity was recorded from the two regions (Fig. $2 D)\left(n=6\right.$ slices). After a combined exposure to $4 \mathrm{mM} \mathrm{K}^{+}$and thrombin, a clear distinction between the two regions was evident: whereas seizure-like activity was still generated in CA3, it was absent in CA1, indicating that CA3 has a lower threshold for generation of seizure by thrombin, which then spreads into CA1, but is not generated there spontaneously.

\section{Discussion}

The present study indicates that thrombin, acting through the PAR1 receptor, exerts two apparently independent actions in the hippocampus that are related to its reported clinical effects. Thrombin produces a slow-rising, long-lasting enhancement of reactivity to afferent stimulation, which shares a downstream mechanism with tetanic LTP, and indeed the two types of potentiation can saturate the ability of the tissue to further increase its response to stimulation. That this potentiation shares a downstream mechanism is also indicated by the ability of an NMDA antagonist, ifenprodil, to attenuate both tetanic and thrombinmediated LTP. Furthermore, APV, an NMDA antagonist, completely blocked thrombin-mediated LTP. The other effect of thrombin, acting at a non-NMDA receptor site, involves lower- ing the threshold for the generation of spontaneous seizure-like activity in the slice. This effect is also dependent on PAR1 receptors, and it is generated only in CA3 region, as are other types of epileptic activities.

The mechanisms activated by thrombin, at the PAR1 receptor site, as well as the physical location of these receptors has been a matter of debate. Some recent evidence indicates that PAR1 receptors are located on glial cells, and may regulate uptake of glutamate in these cells, which may eventually increase the concentration of glutamate at the synaptic cleft (Junge et al., 2004; Lee et al., 2007; Traynelis and Trejo, 2007). Previous studies suggest that thrombin potentiates NMDA receptor function and is likely to reside on neurons (Gingrich et al., 2000). Our results do not provide a clear indication for this locus, but they do indicate that the effect of thrombin is rather slow, which may involve one of several slow-acting mechanisms, such as glutamate uptake into glia, but it will not explain the rather selective action of thrombin on NMDA receptors.

It has been previously hypothesized that PAR1 receptors could have a role in synaptic plasticity (Tsirka et al., 1995; Gingrich et al., 2000; Almonte et al., 2007). However, the lack of clear activators for PAR1 receptors hindered progress in this field. Nevertheless, a number of potential candidates such as plasmin, thrombin, Factor X, and tPA have been suggested (Mizutani et al., 1996; Gingrich et al., 2000; Tomimatsu et al., 2002; Junge et al., 2003). Those molecules could be involved in mediating these receptor functions in terms of synaptic plasticity and development (Gingrich et al., 2000). Particularly, it has been recently shown that PAR1 receptors knock-out mice clearly exhibit impaired memory formation (Almonte et al., 2007), suggesting a potential physiological role for these receptors. In this respect, we found that a short application of a low concentration of PAR1 agonist has the ability to shift the threshold of LTP. This could mean that activators of PAR1 receptors, at low concentrations, could facilitate synaptic plasticity. In pathological situations, the same candidate activators, particularly thrombin, could be present at higher concentrations in the brain, thus leading to the development of pathological features (Gingrich et al., 2000).

The major factors influencing the levels of thrombin in the brain are the levels of active thrombin in the blood, the integrity of the blood-brain barrier (BBB), and the levels of thrombin inhibitors in the brain. The level of active thrombin in the blood is dependent on coagulation, and it may rise more that 1000 -fold during coagulation to reach 50-100 U/ml (Kumar and Chapman, 2007). Clinically, the most dramatic exposure of the brain to thrombin occurs during ICH and SAH (Lin et al., 2003; Claassen et al., 2007). In addition, both ischemia and inflammation raise the levels of thrombin activity in the brain, both influencing brain vascular endothelial cells, thus causing a significant breakdown of the BBB to allow diffusion of large molecules freely into 
neuronal tissue. In ischemic stroke, blood coagulates inside vessels (which comprise 5\% of brain volume), producing thrombin, which gains easy access to the brain through the disrupted BBB. Experimental data indeed show elevated levels of thrombin in acute ischemic models and that specifically blocking the actions of thrombin attenuates neurological deficits (Karabiyikoglu et al., 2004). Once across the BBB, active thrombin is inhibited by endogenous serpins in the brain (Beilin et al., 2001), which are saturated when the levels rise above the buffering capacity of these inhibitors. In inflammatory models such as experimental autoimmune encephalomyelitis, there is widespread deposition of fibrin and activation of thrombin (Paterson et al., 1987; Inaba et al., 2001), as well as greatly elevated levels of thrombin inhibitors (Beilin et al., 2005). The importance of the BBB in protecting against epilepsy is further supported by the observation that in most of the central diseases that cause epilepsy, such as inflammation, tumors, trauma, and stroke, there is breakdown of the BBB.

Long-term effects of both hemorrhagic and ischemic stroke as well as chronic ischemic and inflammatory processes in the brain may involve dementia. It is tempting to speculate that the mechanisms described in the present study explain some of the transient as well as permanent cognitive dysfunction associated with stroke and inflammation. If indeed thrombin saturates the LTPgenerating mechanism in a nonselective manner, it may explain the inability to recover from a cerebrovascular trauma: the saturation of synaptic connectivity by thrombin-activated mechanisms does not allow the brain to use LTP-like plastic processes for either acquisition of new "memories" or adaptation to new motor plans after the insult. Further experiments are needed to explore the longevity of thrombin action in the brain and the ways to overcome thrombin-related cognitive deficits.

Finally, although large concentrations of thrombin may have devastating effects on spontaneous activity of the brain and its ability to undergo plastic changes, small, subthreshold concentrations may in fact be beneficial, and facilitate the ability to express LTP. This possible dual effect of thrombin has been alluded to recently [Luo et al. (2007) and references therein] and is a subject for further experimentation.

\section{References}

Almonte AG, Hamill CE, Chhatwal JP, Wingo TS, Barber JA, Lyuboslavsky PN, David Sweatt J, Ressler KJ, White DA, Traynelis SF (2007) Learning and memory deficits in mice lacking protease activated receptor-1. Neurobiol Learn Mem 88:295-304.

Anderson WW, Collingridge GL (2001) The LTP Program: a data acquisition program for on-line analysis of long-term potentiation and other synaptic events. J Neurosci Methods 108:71-83.

Beilin O, Gurwitz D, Korczyn AD, Chapman J (2001) Quantitative measurements of mouse brain thrombin-like and thrombin inhibition activities. Neuroreport 12:2347-2351.

Beilin O, Karussis DM, Korczyn AD, Gurwitz D, Aronovich R, Hantai D, Grigoriadis N, Mizrachi-Kol R, Chapman J (2005) Increased thrombin inhibition in experimental autoimmune encephalomyelitis. J Neurosci Res 79:351-359.

Camilo O, Goldstein LB (2004) Seizures and epilepsy after ischemic stroke. Stroke 35:1769-1775

Claassen J, Jette N, Chum F, Green R, Schmidt M, Choi H, Jirsch J, Frontera JA, Connolly ES, Emerson RG, Mayer SA, Hirsch LJ (2007) Electrographic seizures and periodic discharges after intracerebral hemorrhage. Neurology 69:1356-1365.

de Haan EH, Nys GM, Van Zandvoort MJ (2006) Cognitive function following stroke and vascular cognitive impairment. Curr Opin Neurol 19:559-564.

Gingrich MB, Traynelis SF (2000) Serine proteases and brain damage-is there a link? Trends Neurosci 23:399-407.

Gingrich MB, Junge CE, Lyuboslavsky P, Traynelis SF (2000) Potentiation of NMDA receptor function by the serine protease thrombin. J Neurosci 20:4582-4595.

Henon H, Pasquier F, Leys D (2006) Poststroke dementia. Cerebrovasc Dis 22:61-70.

Hua Y, Keep RF, Hoff JT, Xi G (2007) Brain injury after intracerebral hemorrhage: the role of thrombin and iron. Stroke 38:759-762.

Inaba Y, Ichikawa M, Inoue A, Itoh M, Kyogashima M, Sekiguchi Y, Nakamura S, Komiyama A, Koh C (2001) Plasma thrombin-antithrombin III complex is associated with the severity of experimental autoimmune encephalomyelitis. J Neurol Sci 185:89-93.

Jensen MS, Yaari Y (1988) The relationship between interictal and ictal paroxysms in an in vitro model of focal hippocampal epilepsy. Ann Neurol 24:591-598.

Jensen MS, Yaari Y (1997) Role of intrinsic burst firing, potassium accumulation, and electrical coupling in the elevated potassium model of hippocampal epilepsy. J Neurophysiol 77:1224-1233.

Junge CE, Sugawara T, Mannaioni G, Alagarsamy S, Conn PJ, Brat DJ, Chan PH, Traynelis SF (2003) The contribution of protease-activated receptor 1 to neuronal damage caused by transient focal cerebral ischemia. Proc Natl Acad Sci USA 100:13019-13024.

Junge CE, Lee CJ, Hubbard KB, Zhang Z, Olson JJ, Hepler JR, Brat DJ, Traynelis SF (2004) Protease-activated receptor-1 in human brain: localization and functional expression in astrocytes. Exp Neurol 188:94-103.

Karabiyikoglu M, Hua Y, Keep RF, Ennis SR, Xi G (2004) Intracerebral hirudin injection attenuates ischemic damage and neurologic deficits without altering local cerebral blood flow. J Cereb Blood Flow Metab 24:159-166.

Kumar V, Chapman JR (2007) Whole blood thrombin: development of a process for intraoperative production of human thrombin. J Extra Corpor Technol 39:18-23.

Lee CJ, Mannaioni G, Yuan H, Woo DH, Gingrich MB, Traynelis SF (2007) Astrocytic control of synaptic NMDA receptors. J Physiol 581:1057-1081.

Lee KR, Drury I, Vitarbo E, HoffJT (1997) Seizures induced by intracerebral injection of thrombin: a model of intracerebral hemorrhage. J Neurosurg 87:73-78.

Lee SY, Haydon PG (2007) Astrocytic glutamate targets NMDA receptors. J Physiol 581:887-888.

Li R, Huang FS, Abbas AK, Wigstrom H (2007) Role of NMDA receptor subtypes in different forms of NMDA-dependent synaptic plasticity. BMC Neurosci 8:55.

Lin CL, Dumont AS, Lieu AS, Yen CP, Hwang SL, Kwan AL, Kassell NF, Howng SL (2003) Characterization of perioperative seizures and epilepsy following aneurysmal subarachnoid hemorrhage. J Neurosurg 99:978-985.

Liu L, Wong TP, Pozza MF, Lingenhoehl K, Wang Y, Sheng M, Auberson YP, Wang YT (2004) Role of NMDA receptor subtypes in governing the direction of hippocampal synaptic plasticity. Science 304:1021-1024.

Luo W, Wang Y, Reiser G (2007) Protease-activated receptors in the brain: receptor expression, activation, and functions in neurodegeneration and neuroprotection. Brain Res Rev 56:331-345.

Maggio N, Segal M (2007) Striking variations in corticosteriod modulation of long term potentiation along the septotemporal axis of the hippocampus. J Neurosci 27:5757-5765.

Mizutani A, Saito H, Matsuki N (1996) Possible involvement of plasmin in long-term potentiation of rat hippocampal slices. Brain Res 739:276-281.

Paterson PY, Koh CS, Kwaan HC (1987) Role of the clotting system in the pathogenesis of neuroimmunologic disease. Fed Proc 46:91-96.

Sun DA, Sombati S, DeLorenzo RJ (2001) Glutamate injury-induced epileptogenesis in hippocampal neurons: an in vitro model of strokeinduced "epilepsy." Stroke 32:2344-2350.

Tomimatsu Y, Idemoto S, Moriguchi S, Watanabe S, Nakanishi H (2002) Proteases involved in long-term potentiation. Life Sci 72:355-361.

Traynelis SF, Dingledine R (1988) Potassium-induced spontaneous electrographic seizures in the rat hippocampal slice. J Neurophysiol 59:259-276.

Traynelis SF, Trejo J (2007) Protease-activated receptor signaling: new roles and regulatory mechanisms. Curr Opin Hematol 14:230-235.

Tsirka SE, Gualandris A, Amaral DG, Strickland S (1995) Excitotoxininduced neuronal degeneration and seizure are mediated by tissue plasminogen activator. Nature 377:340-344.

Xi G, Reiser G, Keep RF (2003) The role of thrombin and thrombin receptors in ischemic, hemorrhagic and traumatic brain injury: deleterious or protective? J Neurochem 84:3-9. 